\title{
The Hyperboloidal Numerical Evolution of a Good-Bad-Ugly Wave Equation
}

\author{
Edgar Gasperín ${ }^{1}$, Shalabh Gautam ${ }^{2}$, David Hilditch ${ }^{1}$ and Alex Vañó-Viñuales ${ }^{1,3}$ \\ ${ }^{1}$ CENTRA, Departamento de Física, Instituto Superior Técnico IST, \\ Universidade de Lisboa UL, Avenida Rovisco Pais 1, 1049 Lisboa, Portugal, \\ ${ }^{2}$ Inter-University Centre for Astronomy and Astrophysics, Post Bag 4, Ganeshkhind, Pune 411007, India, \\ ${ }^{3}$ School of Physics and Astronomy, Cardiff University, \\ Queen's Buildings, The Parade, CF24 3AA, Cardiff, United Kingdom
}

(Dated: September 27, 2019)

\begin{abstract}
One method for the numerical treatment of future null-infinity is to decouple coordinates from the tensor basis and choose each in a careful manner. This dual-frame approach is hampered by logarithmically divergent terms that appear in a naive choice of evolved variables. Here we consider a system of wave equations that satisfy the weak-null condition and serve as a model system with similar nonlinearities to those present in the Einstein field equations in generalized harmonic gauge. We show that these equations can be explicitly regularized by a nonlinear change of variables. Working in spherical symmetry, a numerical implementation of this model using compactified hyperboloidal slices is then presented. Clean convergence is found for the regularized system. Although more complicated, it is expected that general relativity can be treated similarly.
\end{abstract}

\section{INTRODUCTION}

For applications in gravitational wave astronomy it is desirable to consider generic asymptotically flat spacetimes and compute, using the methods of numerical relativity, waveforms at infinity. This is a long-standing open problem. Following Penrose [1] and Friedrich [2, 3], a natural strategy is to work on compactified hyperboloidal slices, see 4 or 5 for reviews. One proposal for this problem [6] is to use a dual-frame (DF) [7] approach. In this setting the Einstein field equations (EFEs) are written using a generalized harmonic tensor basis, and solved in the aforementioned hyperboloidal coordinates. The key necessary requirement for this approach to work is that certain derivatives of outgoing radial coordinate lightspeeds have suitable decay. Recently it was shown [8] that this lightspeed condition is related to the weak-null condition [9], an important structural condition for small-data global existence to nonlinear wave-equations, see 10 for further discussion of the latter. Using the notion of the asymptotic system, it was furthermore heuristically shown that even in a pure freeevolution setup, by making a suitable addition of constraints to the field equations, the lightspeed condition can be satisfied within the scheme of [6].

The equations of motion in [6] were constructed to avoid the presence of formally singular terms, but the simplest choice of variables leaves some variables like $(\ln R) / R$, near null-infinity. Such terms could be problematic for numerics. Therefore, going beyond the bare-bones scheme of [6], we would like to obtain regular equations for regular unknowns that are not required to vanish at future-null infinity. The aim here is to show that this can be achieved in a nonlinear good-bad-ugly wave equation model whose nonlinearities mimic those present in the formulation of the EFEs given in 8. This builds towards a full-blown regularization of general relativity (GR) in harmonic gauge, which will be presented elsewhere, and may serve as an alternative to the con- formal field equations [1]. We present an implementation with a battery of tests indicating that reliable, convergent, results can be obtained using our regularization technique.

\section{A GOOD-BAD-UGLY MODEL}

In this section we present our model problem and give a new strategy for regularization on hyperboloidal slices that exploits the nonlinear structure of the field equations. We start in section $\llbracket \mathrm{A}$ with the model in second order form and then reduce to first order in section IIB. The regularization is implemented in section IIC and finally give a form of the asymptotic system in compactified hyperboloidal coordinates in section IID.

\section{A. Second order form}

In our previous study 8 we found that in harmonic gauge the field equations of GR can be divided into three categories, which we call the good, the bad and the ugly. This categorization is made by deriving from the original set of equations an associated asymptotic system, which in turn can be used to predict decay rates of the variables near null-infinity. The good equations are those for whom the asymptotic system indicates fall-off identical to that of the standard wave-equation. The bad equations are those whose solutions decay slower than that. In the case of GR in harmonic gauge this can be restricted to a logarithmic loss in a single equation. The ugly equations are those associated with the constraints. These equations can be manipulated by addition of the constraints. This can give messier expressions, but ultimately results in fall-off faster than that of the wave equation. Let $\left(\mathbb{R}^{4}, m_{a b}\right)$ denote the Minkowski spacetime and metric and denote its corresponding Levi-Civita connection as $\nabla$. The model equation to be studied in this 
paper,

$$
\square g=0, \quad \square b=\left(\partial_{T} g\right)^{2}, \quad \square u=\frac{2}{\chi} \partial_{T} u,
$$

consists of a system of wave equations that ape this structure. Here $\square$ denotes the d'Alembert operator in the Minkowski spacetime and $\chi \simeq R$ where $R^{2}=\delta_{i j} X^{\underline{i}} X^{j}$. In these expressions $X^{\underline{\mu}}=\left(T, X^{\underline{i}}\right)$, with $\mu \in\{\underline{0,1}, 2,3\}$ and $\underline{i} \in\{1,2,3\}$, denote global inertial Cartesian coordinates. We use the symbol $\simeq$ to represent equality at large radius up to error terms that decay faster in $R$ than those displayed in the expression. For instance $f=R^{-1}+O\left(R^{-(1+\delta)}\right)$ with $\delta>0$ will be written simply as $f \simeq R^{-1}$. The model (1) is an example of a system of wave equations satisfying the weak null condition. To understand what this means, one has to derive the asymptotic system mentioned above.

Here we sketch the construction as given in [9]. In section IID we give an alternative method to obtain the asymptotic system that uses hyperboloidal coordinates directly. We begin by introducing shellcoordinates $X^{\mu^{\prime}}=\left(T, R, \theta^{A}\right)$ where $\theta^{A}$ with $A \in\{1,2\}$ denote arbitrary coordinates on $\mathbb{S}^{2}$, whose specific form will be fixed later, and then defining the rescaled variables $\mathcal{G}=R g, \mathcal{B}=R b$ and $\mathcal{U}=R u$. Now, consider the coordinate change $\left(T, R, \theta^{A}\right) \rightarrow\left(v, s, \theta^{A}\right)$ where $v=T-R$ and $s=\ln R$. Rewriting the system in terms of $\mathcal{G}, \mathcal{B}$ and $\mathcal{U}$ and formally equating the terms with coefficients $1 / R^{2}$ gives,

$$
2 \partial_{s} \partial_{v} \mathcal{B}=-\left(\partial_{v} \mathcal{G}\right)^{2}, \quad \partial_{s} \partial_{v} \mathcal{G}=0, \quad \partial_{s} \partial_{v} \mathcal{U}=-\partial_{v} \mathcal{U}
$$

This is the asymptotic system associated with equation (1). Observe that the second equation in (2) implies that $\partial_{v} \mathcal{G}$ depends only on $v$. Consequently, one can integrate the first equation of 2 in $s$ and conclude that $\partial_{v} \mathcal{B}=-\frac{s}{2}\left(\partial_{v} \mathcal{G}\right)^{2}$. Hence in the asymptotic approximation $\mathcal{B}=-\frac{1}{2} \ln (R) \int_{v_{\star}}^{v}\left(\partial_{\bar{v}} \mathcal{G}\right)^{2} \mathrm{~d} \bar{v}$. From the third equation one concludes that $\partial_{v} \mathcal{U}=\left.\frac{1}{R}\left(\partial_{v} \mathcal{U}\right)\right|_{s_{\star}}$. Integrating this gives $\mathcal{U}=\left.\frac{1}{R} \int_{v_{\star}}^{v}\left(\partial_{v} \mathcal{U}\right)\right|_{s_{\star}} \mathrm{d} \bar{v}$. So the asymptotic system for $u$ indicates that one could have taken a further power of $1 / R$ in the definition of the rescaled variable $\mathcal{U}$. [The right-hand side of the equation for $u$ was of course chosen precisely for this purpose]. Nevertheless, observe that given a function $m=m(\theta, \varphi)$ such that $4 m=0$, where $\not \subset$ denotes the Laplacian on $\mathbb{S}^{2}$, one has that $m / R$ is a solution to any of the equations in (1). In the remainder we will discard these solutions. In other words, we consider fields $u, g$ and $b$ where we have subtracted any static solutions of this form.

A system of wave equations is said to satisfy the weak null condition if its asymptotic system admits global solutions which grow at most exponentially in $s$. Recall that a quadratic form $N^{\mu \nu} \partial_{\mu} \phi \partial_{\nu} \phi$ is a null-form if it vanishes upon replacement of $\partial_{\mu} \phi$ with $\xi_{\mu}$ where $\xi^{\mu}$ is a null vector. A wave equation is said to satisfy the classical null condition 1214 if its nonlinearity can be written as a sum of null-forms. A system of quasilinear wave equations satisfying the classical null condition trivially satisfies the weak null condition. One naive question that arises from this analysis is whether the logarithmic loss predicted by the asymptotic equation for $\mathcal{B}$ is somehow tied to the choice of the coordinate system $\left(v, s, \theta^{A}\right)$. In other words, is the logarithmic loss only present due to the choice $s(R)=\ln (R)$, or could better coordinates give a sharper result? In Section IID a new version of the above analysis is given. In this new approach a compactified radial coordinate is used instead of the aforementioned stretch $R=e^{s}$. Identical results are obtained, indicating that the outcome is robust against coordinate changes.

The model equation (1) is an example of a system of wave equations that fails to satisfy the classical null condition, but does satisfy the weak null condition. We call the system of equations (1) the semilinear good-bad-ugly model since $g$ is a field (the good) that satisfies an equation that fulfills the classical null condition while $b$ (the bad) is a field which is responsible for the failure of the system to satisfy the classical null condition. Since the Einstein field equations in harmonic gauge satisfy the weak null condition the good-bad subsystem alone constitutes a simple toy model to be implemented numerically in the dual foliation framework [6, 7] which en-captures this feature. This subsystem is furthermore the simplest toy model in which one can test regularization strategies for the fields analogous to the bad metric components, as they appear in generalized harmonic gauge in the EFE. The addition of the variable $u$ (the ugly) to the good-bad subsystem arises as a model for the type of equations that appear in the evolution equations if one adds certain multiples of the harmonic constraints to the EFE. As discussed in [8] such an addition is required for a successful numerical implementation of the hyperboloidal initial value problem using the DF formalism. The equations in expression (1) almost decouple, naturally we could have analyzed a more complicated system of equations satisfying the weak null condition. Adding null-forms for instance would leave the asymptotic system unchanged, but we instead want to consider the simplest good-badugly model with the properties described above.

\section{B. First order reduction of the model}

Let $\left(\mathbb{S}^{2}, q_{a b}\right)$ denote the unit two-sphere with the standard metric and represent by $\not$ the associated Levi-Civita connection. The shell coordinates $X^{\mu^{\prime}}=$ $\left(T, R, \theta^{A}\right)$ will be regarded, in the language of the DF formalism [7] as the uppercase coordinates.

For numerical implementation it is convenient to perform a first order reduction of the model. To that end, we make use of the usual $3+1$ decomposition. Let $\Sigma_{T}$ be the hypersurface determined by the condition $T=$ const., $N^{a}$ denotes the timelike unit normal to this surface and define the projector $\gamma_{a b}$ as ${ }^{(N)} \gamma_{a b}=m_{a b}+N_{a} N_{b}$. We then 
define the time reduction variable $g_{\pi}$ by $g_{\pi}=-\partial_{T} g$ and introduce a spatial covector $g_{a}$ and denote as $g_{R}$ and $g_{A}$ the components of $g_{a}$ respect to $\left(\left(\partial_{R}\right)^{a},\left(\partial_{A}\right)^{a}\right)$. The reduction constraints are

$$
{ }^{(g)} \mathcal{C}_{a} \equiv \gamma_{a}{ }^{b} \nabla_{b} g-g_{a} .
$$

Analogous definitions are made for the $b$ and $u$ fields. Using this notation, the semilinear good-bad-ugly model system can be written in first order form as follows. For the goods we have,

$$
\begin{aligned}
\partial_{T} g & =-g_{\pi}, \\
\partial_{T} g_{R} & =-\partial_{R} g_{\pi}+\gamma_{2}\left(-g_{R}+\partial_{R} g\right), \\
\partial_{T} g_{A} & =-\partial_{A} g_{\pi}+\gamma_{2}\left(-g_{A}+\partial_{A} g\right), \\
\partial_{T} g_{\pi} & =-\partial_{R} g_{R}-\frac{1}{R^{2}} q^{A B} \nabla_{A} g_{B}-\frac{2}{R} g_{R},
\end{aligned}
$$

while for the bads the equations are

$$
\begin{aligned}
\partial_{T} b & =-b_{\pi}, \\
\partial_{T} b_{R} & =-\partial_{R} \pi+\gamma_{2}\left(-b_{R}+\partial_{R} b\right), \\
\partial_{T} b_{A}= & -\partial_{A} b_{\pi}+\gamma_{2}\left(-b_{A}+\partial_{A} b\right), \\
\partial_{T} b_{\pi} & =g_{\pi}^{2}-\partial_{R} b_{R}-\frac{1}{R^{2}} q^{A B} \nabla_{A} b_{B}-\frac{2}{R} b_{R},
\end{aligned}
$$

and finally for the uglies, the evolution equations read

$$
\begin{aligned}
\partial_{T} u & =-u_{\pi}, \\
\partial_{T} u_{R} & =-\partial_{R} u_{\pi}+\gamma_{2}\left(-u_{R}+\partial_{R} u\right), \\
\partial_{T} u_{A} & =-\partial_{A} u_{\pi}+\gamma_{2}\left(-u_{A}+\partial_{A} u\right), \\
\partial_{T} u_{\pi} & =-\frac{2}{\chi} u_{\pi}-\partial_{R} u_{R}-\frac{1}{R^{2}} q^{A B} \nabla_{A} u_{B}-\frac{2}{R} u_{R} .
\end{aligned}
$$

Here $\gamma_{2}$ is a freely prescribable scalar function of the coordinates. The definition of the time reduction variables is encoded in the evolution equations $4 \mathrm{a}, 4 \mathrm{e}$ and $4 \mathrm{i}$. Setting $\gamma_{2}=0$ one sees that the evolution equations (4b), (4f) and (4j) were obtained from the no-torsion condition $\left[\nabla_{a}, \nabla_{b}\right] \phi=0$, with $\phi=g, b, u$. Observe that the term next to $\gamma_{2}$ corresponds to the reduction constraints. These terms are introduced to mitigate constraint violation in free evolution schemes. Their addition affects the principal part of the equations, but nevertheless the system is symmetric hyperbolic for any choice of the formulation parameter $\gamma_{2}$ 15. Finally, expressing (1) using the reduction variables renders the evolution equations $(4 \mathrm{~d})$, (4h) and (4l).

\section{Evolution equations for the rescaled variables}

In this section we rescale the variables and derive evolution equations for them. In the construction of the asymptotic system we rescaled all of the fields identically. As examined in 8, this leads to a first order version of the asymptotic system discussed in section II A. Nevertheless, evolving the first order variables analogous to $\mathcal{G}, \mathcal{B}$ and $\mathcal{U}$ is not convenient for numerical applications because $\mathcal{B}$ is expected to diverge as $\ln (R)$ and $\mathcal{U}$ to decay as $1 / R$ near null-infinity. Ideally we want regular equations for variables for which one expects a regular behavior and a finite, potentially non-zero, limit at null infinity. The latter suggests performing a different rescaling for the good, the bad and the ugly fields. In this section we discuss how to do this in such a way that the evolution equations for the rescaled variables in hyperboloidal coordinates $x^{\mu}=\left(t, r, \theta^{A}\right)$, corresponding to the lowercase coordinates in the nomenclature of the DF formalism, are regular.

Asymptotic system primer: We denote the outgoing and incoming null vectors by $L$ and $\underline{L}$ respectively,

$$
L^{a}=\left(\partial_{T}\right)^{a}+\left(\partial_{R}\right)^{a}, \quad \underline{L}^{a}=\left(\partial_{T}\right)^{a}-\left(\partial_{R}\right)^{a},
$$

and define

$$
g^{+}=-g_{\pi}+g_{R}, \quad g^{-}=-g_{\pi}-g_{R},
$$

and analogous quantities for the fields $b$ and $u$. Observe that the ' + ' and ' - ' variables correspond to the $L$ and $\underline{L}$ derivatives of the original fields, or, in other words, the characteristic variables of the system (4) with $\gamma_{2}=0$. Substituting $g_{\pi}, g_{R}, b_{\pi}, b_{R}, u_{\pi}$ and $u_{R}$ written in terms of the ' + ' and ' - ' variables into equations (4a)- 41) one obtains a set of evolution equations for the ' + ', ' - ' and ' $A$ ' variables for $g, b$ and $u$. The next step is to rescale and choose the variables to evolve. Since there is a large freedom in doing this in practice, to explain the rationale behind the choice that we make, first, let us examine the standard rescaling suggested by the discussion of the asymptotic system of Section IIA. We use a schematic notation and let $\phi$ represent $g, b, u$. Likewise $\phi_{A}$ encodes the angular variables and, as in equation $(6), \phi^{ \pm}$the characteristic variables. Similarly we denote by $\Phi, \Phi_{A}, \Phi^{ \pm}$the rescaled variables $\mathcal{G}, \mathcal{G}_{A}, \mathcal{G}^{ \pm}, \mathcal{B}, \mathcal{B}_{A}, \mathcal{B}^{ \pm}, \mathcal{U}, \mathcal{U}_{A}, \mathcal{U}^{ \pm}$. With this notation at hand, the following expressions should be regarded as the 'basic' rescaling,

$$
\Phi^{+}=R^{2} \phi^{+}, \Phi^{-}=R \phi^{-}, \Phi=R \phi, \Phi_{A}=R \phi_{A} .
$$

This corresponds, in terms of the original fields to taking the derivative first and rescaling afterwards, namely

$$
\Phi^{+}=R^{2} L \phi, \quad \Phi^{-}=R \underline{L} \phi, \quad \Phi=R \phi, \quad \Phi_{A}=R \partial_{A} \phi .
$$

Following the procedure described in [8] and, for simplicity of the presentation, taking $\gamma_{2}=0$, one obtains the following first order asymptotic system:

$$
\begin{aligned}
\partial_{v} \mathcal{G}^{+} & =-\frac{1}{2} \mathcal{G}^{-}, & \partial_{v} \mathcal{B}^{+} & =-\frac{1}{2} \mathcal{B}^{-}-\frac{1}{8}\left(\mathcal{G}^{-}\right)^{2}, \\
\partial_{s} \mathcal{G}^{-} & =0, & \partial_{s} \mathcal{B}^{-} & =-\frac{1}{4}\left(\mathcal{G}^{-}\right)^{2}, \\
\partial_{v} \mathcal{U}^{+} & =-\mathcal{U}^{-}, & \partial_{v} \Phi & =\frac{1}{2} \Phi^{-}, \\
\partial_{s} \mathcal{U}^{-} & =-\mathcal{U}^{-} & \partial_{v} \Phi_{A} & =\frac{1}{2} \partial_{A} \Phi^{-} .
\end{aligned}
$$

Since we have chosen here the same rescaling for all the variables regardless of the equation they satisfy it is natural that their asymptotic equations differ. Nonetheless, 
in the following we will discuss how to exploit the information provided by the asymptotic system $(9)$ in order to obtain optimal definitions for the rescaled variables.

First notice that, although the decay of the good fields cannot be improved, the asymptotic system for these fields can be written in a slightly simpler way by considering the following variables

$$
G^{+}=\mathcal{G}^{+}+\mathcal{G}, \quad G^{-}=\mathcal{G}^{-}, \quad G=\mathcal{G}, \quad G_{A}=\mathcal{G}_{A} .
$$

Then, the asymptotic system for the good variables reads,

$$
\begin{aligned}
\partial_{v} G^{+} & =0, & & \partial_{s} G^{-}=0, \\
\partial_{v} G & =\frac{1}{2} G^{-}, & \partial_{v} G_{A} & =\frac{1}{2} \partial_{A} G^{-} .
\end{aligned}
$$

In terms of the original unrescaled field, the latter change corresponds to the following choice of variables.

$$
G^{+}=R L(R g), G^{-}=R \underline{L g}, G=R g, G_{A}=R \partial_{A} g .
$$

Henceforth, we regard the form of the equations (11) as the prototype for the asymptotic system for a set of equations that have the same asymptotics as that of the homogeneous wave equation.

In order to find the optimal redefinition for the rescaled uglies, first recall that, as already discussed in Section II A, the in-homogeneity in the equation for the ugly was designed so that the field $u$ decays one order $1 / R$ faster than $g$. This property can be read off directly from the asymptotic equation $\partial_{s} \mathcal{U}^{-}=-\mathcal{U}^{-}$. This suggests rescaling the fields with one extra power of $1 / R$ of that of the basic rescaling (7), furthermore, some experimentation reveals that by defining

$$
\begin{aligned}
U^{+} & =R\left(\mathcal{U}^{+}+2 \mathcal{U}\right), & U^{-} & =R \mathcal{U}^{-}, \\
U & =R \mathcal{U}, & U_{A} & =R \mathcal{U}_{A},
\end{aligned}
$$

the asymptotic equations for these variables are identical to (11) if we-formally replace $G^{ \pm}$and $G$ by $U^{ \pm}$and $U$ respectively. Written in terms of the raw ugly field we have,

$$
\begin{aligned}
U^{+} & =R L\left(R^{2} u\right), & & U^{-}=R^{2} \underline{L} u, \\
U & =R^{2} u, & U_{A} & =R^{2} \partial_{A} u .
\end{aligned}
$$

For the bad fields we make the following nonlinear change of variables

$$
\begin{aligned}
B^{+} & =\mathcal{B}^{+}+\mathcal{B}+\frac{1}{8} \eta, & B^{-} & =\mathcal{B}^{-}+\frac{1}{4} s \partial_{v} \eta, \\
B & =\mathcal{B}+\frac{1}{8} s \eta, & B_{A} & =\mathcal{B}_{A}+\frac{1}{8} \partial_{A} \eta .
\end{aligned}
$$

where $\eta$ is an auxiliary variable whose evolution equation has to be chosen in such a way that in the asymptotic limit it reduces to $\partial_{v} \eta=\left(G^{-}\right)^{2}$. With these definitions, a direct calculation shows that the asymptotic system for the $B$-fields, which we call the reformed bads is identical to that of the goods under replacement of $G^{ \pm}$ and $G$ by $B^{ \pm}$and $B$ respectively in equation (11). The price to pay for this regularization is the introduction of a new variable $\eta$ which, as in the asymptotic system, encodes $\int_{v_{\star}}^{v}\left(\partial_{\bar{v}} G\right)^{2} \mathrm{~d} \bar{v}$. Although $\eta$ is defined as an integral it will satisfy a local equation of motion. The change of variables 15 can be written in terms of the raw bad field as

$$
\begin{aligned}
B^{+} & =R L\left(R b+\frac{1}{8} s \eta\right), & B^{-} & =R \underline{L}(b)+\frac{1}{8} s \underline{L} \eta, \\
B & =R b+\frac{1}{8} s \eta, & B_{A} & =R \partial_{A} b+\frac{1}{8} s \partial_{A} \eta .
\end{aligned}
$$

This regularization strategy can be thought of as "subtracting" the $\ln (R)$ part of the asymptotic solution for $\mathcal{B}$. An alternative regularization strategy is, instead, to "divide" by $\ln (R)$. The disadvantage of the latter, arguably simpler option, is that it generates slowly decaying $1 / \ln (R)$ terms in the evolution equations. We have implemented this regularization also, but find that these slowly decaying terms prevent the code from converging, and so do not discuss the method further.

Complete evolution equations in hyperboloidal coordinates: The foregoing discussion already demonstrates how to choose the rescaled variables in order to have regular equations at $\mathscr{I}^{+}$. Nevertheless, as will be elaborated further in the remainder of this section, care is needed at the origin $R=0$ if we wish to evolve numerically the good-bad-ugly system in spherical symmetry. Taking this into account, a suitable choice for the rescaled variables is, for the goods,

$$
\begin{aligned}
G^{+} & =\chi^{2} g^{+}+R g, & & G^{-}=\chi g^{-}, \\
G & =\chi g, & G_{A} & =\chi g_{A},
\end{aligned}
$$

for the bads,

$$
\begin{aligned}
B^{+} & =\chi^{2} b^{+}+R b+\frac{1}{8} \chi^{-1} R \eta, & B & =\chi b+\frac{1}{8}(\xi-1) \eta, \\
B^{-} & =\chi b^{-}+\frac{1}{4}(\xi-1) \partial_{T} \eta, & B_{A} & =\chi b_{A}+\frac{1}{8}(\xi-1) \eta_{A},
\end{aligned}
$$

and finally, for the uglies,

$$
\begin{aligned}
U^{+} & =\chi^{3} u^{+}+2 \chi R u, & U^{-} & =\chi^{2} u^{-}, \\
U & =\chi^{2} u, & U_{A} & =\chi^{2} u_{A},
\end{aligned}
$$

where $\chi=\chi(R)$ and $\xi \equiv \ln \chi$. The even function $\chi$ is to be chosen such that $\chi(0)=1$ and $\chi \simeq R$ at large radii. This ensures that, asymptotically, the change of variables is that of equations $(10),(13)$ and $(15)$, while at the origin the transformation reduces to the identity. Additionally, we have introduced new variables $\eta$ and $\eta_{A}$, the latter encoding the angular derivatives of the former. Thus, associated to $\eta_{A}$ we introduce the constraint

$$
{ }^{(\eta)} C \equiv \nabla_{A} \eta-\eta_{A}
$$

Once the evolution equation for $\eta$ is chosen, the equation for $\eta_{A}$ can be obtained exploiting the no-torsion condition $\left[\nabla_{a}, \nabla_{b}\right] \eta=0$. From the previous discussion we know that the evolution equation for $\eta$ has to be chosen such that it asymptotically reduces to $\partial_{v} \eta=\left(G^{-}\right)^{2}$ in order for our regularization strategy to work. A simple 
choice, to which we adhere from this point onward, is to set

$$
\partial_{T} \eta=4 R^{2} g_{\pi}^{2}
$$

Expressed in rescaled variables this reads,

$$
\partial_{T} \eta=\frac{R^{2}}{\chi^{2}}\left(G^{-}+\frac{1}{\chi} G^{+}-\frac{R}{\chi^{2}} G\right)^{2} .
$$

Using the no-torsion condition as described before and equation 20 we obtain the following for $\eta_{A}$,

$$
\begin{aligned}
\partial_{T} \eta_{A}= & \frac{2 R^{2}}{\chi^{2}}\left(G^{-}+\frac{1}{\chi} G^{+}-\frac{R}{\chi^{2}} G\right) \times \\
& \left(\not_{A} G^{-}+\frac{1}{\chi} \not_{A} G^{+}-\frac{R}{\chi^{2}} G_{A}\right) .
\end{aligned}
$$

Expressing the evolution equations (4) in terms of the rescaled variables as defined around equation (17) is a straightforward but cumbersome calculation. The reason for the latter is twofold: the change of variables (17) was designed so that at the origin the rescaled variables reduce to the unrescaled characteristic variables $\phi^{+}, \phi^{-}, \phi$ and $\phi_{A}$. To do so, we had to introduce functions such as $\chi$ instead of simply $R$ or $\xi-1$ instead of just $\ln (R)$, that when pushed through the change of variables produce several non-principal terms. Second, although we expect to obtain the simplest possible expression for the asymptotic equation under this choice of variables, due to the extra term added in the definition of the + fields, we do not get simple advection equations. Compare for instance the definition for $G^{+}$and $\mathcal{G}^{+}$in equations $(7)$ and $(10)$. Nevertheless, we improve this situation by adding multiples of the constraints appropriately to absorb these extra terms and thus reduce the system to a set of advection equations near infinity. We omit the details of this computation. We know that the original equations are symmetric hyperbolic, but what of the modified set? After changing variables, we end up with a system which takes a standard first order symmetric hyperbolic form for all of the fields except the reformed bads $B, B^{+}, B^{-}, B_{A}$, plus $\eta$ and $\eta_{A}$, each of which look like a system that would be trivially symmetric hyperbolic if the derivative coupling to the rescaled good fields could be dropped. This additional coupling can be treated as non-principal however, by noting that the full system can be consistently evolved under the assumption that the good fields are one degree of regularity better behaved (in suitable Sobolev spaces) than the reformed bads. Although we have identified the leading behavior of the fields via the asymptotic system analysis, a deeper understanding of the solution could perhaps be achieved by obtaining a hierarchical set of "higher order asymptotic systems" determining the subleading terms in the solution. This is left for future work. To express the evolution equations in their final form, we define radially compactified hyperboloidal coordinates $\left(t, r, \theta^{A}\right)$ related to $\left(T, R, \theta^{A}\right)$ via,

$$
T=t+H(R(r)), \quad R=R(r),
$$

and let $H^{\prime}=d H / d R$ and $R^{\prime}=d R / d r$. A direct computation shows that the above evolution equations for the rescaled variables in the $\left(t, r, \theta^{A}\right)$ coordinates read as,

$$
\begin{aligned}
\partial_{t} G^{+}= & -c_{-}^{r} \partial_{r} G^{+}-\mathcal{A}^{-} q^{A B} \not_{A} G_{B}+S_{G^{+}} \\
\partial_{t} G^{-}= & -c_{+}^{r} \partial_{r} G^{-}-\gamma_{2} c_{+}^{r} \partial_{r} G+\mathcal{A}^{+} q^{A B} \nabla_{A} G_{B}+S_{G^{-}} \\
\partial_{t} G_{A}= & \frac{1}{2} \partial_{A}\left(G^{-}+\frac{1}{\chi} G^{+}\right)+\gamma_{2} \partial_{A} G-\gamma_{2} G_{A} \\
& -\frac{R}{2 \chi^{2}} G_{A} \\
\partial_{t} G= & \frac{1}{2} G^{-}-\frac{R}{2 \chi^{2}} G+\frac{1}{2 \chi} G^{+}
\end{aligned}
$$

for the goods, whilst for the bads we have,

$$
\begin{aligned}
\partial_{t} B^{+}= & -c_{-}^{r} \partial_{r} B^{+}-\mathcal{A}^{-} q^{A B} \not_{A} B_{B}+F_{\eta}^{-} \partial_{r} \eta \\
& +\frac{1}{8}(\xi-1) \mathcal{A}^{-} q^{A B} \not_{A} \eta_{B}+S_{B^{+}}, \\
\partial_{t} B^{-}= & -c_{+}^{r} \partial_{r} B^{-}-\gamma_{2} c_{+}^{r} \partial_{r} B+\mathcal{A}^{+} q^{A B} \not_{A} B_{B}+F_{\eta}^{+} \partial_{r} \eta \\
& -\frac{1}{8}(\xi-1) \mathcal{A}^{+} q^{A B} \not_{A} \eta_{B}+M \partial_{r} G^{+}+\gamma_{2} J \partial_{r} G \\
& +K q^{A B} \not_{A} G_{B}+S_{B^{-}}, \\
\partial_{t} B_{A}= & \frac{1}{2} \partial_{A}\left(B^{-}+\frac{1}{\chi} B^{+}\right)+\frac{1}{8} \gamma_{2}(\xi-1) \partial_{A} \eta+\gamma_{2} \partial_{A} B \\
& -\left(\gamma_{2}+\frac{R}{2 \chi^{2}}\right) B_{A}+F_{\eta}^{\theta} \eta_{A}, \\
\partial_{t} B= & \frac{1}{2} B^{-}-\frac{R}{2 \chi^{2}} B+\frac{1}{2 \chi} B^{+}+\frac{R}{16 \chi^{2}}(\xi-2) \eta .
\end{aligned}
$$

Note here the aforementioned derivative coupling to the rescaled good fields, and the advection-equation like form of both 23 and $(24)$ near $\mathscr{I}^{+}$. Next for the uglies we get,

$$
\begin{aligned}
\partial_{t} U^{+} & =-c_{-}^{r} \partial_{r} U^{+}-\mathcal{A}^{-} q^{A B} \nabla_{A} U_{B}+S_{U^{+}}, \\
\partial_{t} U^{-} & =-c_{+}^{r} \partial_{r} U^{-}-\gamma_{2} c_{+}^{r} \partial_{r} U+\mathcal{A}^{+} q^{A B} \nabla_{A} U_{B}+S_{U^{-}}, \\
\partial_{t} U_{A} & =\frac{1}{2} \partial_{A}\left(U^{-}+\frac{1}{\chi} U^{+}\right)+\gamma_{2} \partial_{A} U-\gamma_{2} U_{A}-\frac{R}{\chi^{2}} U_{A}, \\
\partial_{t} U & =\frac{1}{2} U^{-}-\frac{R}{2 \chi^{2}} U+\frac{1}{2 \chi} U^{+},
\end{aligned}
$$

and for the auxiliary variable,

$$
\partial_{t} \eta=\frac{R^{2}}{\chi^{2}} P^{2} \quad \partial_{t} \eta_{A}=\frac{2 R^{2}}{\chi^{2}} P \partial_{A} P,
$$

where the various coefficients in $23-26$ are given by,

$$
\begin{aligned}
\alpha_{+} & =1, & \alpha_{-} & =\chi, \\
\beta_{+} & =0, & \beta_{-} & =1, \\
c_{ \pm}^{r} & =\frac{ \pm 1}{\left(1 \mp H^{\prime}\right) R^{\prime}}, & \mathcal{A}^{ \pm} & =\frac{R^{\prime}}{R^{2}} \alpha_{ \pm} c_{ \pm}^{r}, \\
M & =\frac{R^{\prime} R^{2}}{\chi^{3}}(\xi-1) c_{-}^{r} c_{+}^{r} P, & K & =\frac{R^{\prime}}{R^{2}} \chi M, \\
J & =\frac{\chi\left(1-R^{\prime} c_{+}^{r}\right)}{R^{\prime} c_{+}^{r}} M, & P & =G^{-}+\frac{1}{\chi} G^{+}-\frac{R}{\chi^{2}} G,
\end{aligned}
$$

and,

$$
\begin{aligned}
F_{\eta}^{ \pm} & =\frac{c_{ \pm}}{8}\left(\frac{R}{\chi} \beta_{ \pm}+\gamma_{2}(\xi-1) \alpha_{ \pm}\right), \\
F_{\eta}^{\theta} & =\frac{1}{8}\left(\gamma_{2}(\xi-1)+\frac{R}{2 \chi^{2}}(\xi-2)\right) .
\end{aligned}
$$

The remaining lower order terms contained in $S_{G^{ \pm}}, S_{B^{ \pm}}$, and $S_{U^{ \pm}}$are given in detail in appendix $\mathrm{A}$. In view of the 
definition of the reduction constraints in equation (3) and the definition of the rescaled variables (17), we define the rescaled reduction constraints as

$$
\begin{array}{ll}
{ }^{(G)} \mathbb{C}_{R} \equiv \chi^{2}\left({ }^{(g)} \mathcal{C}_{R}\right), & { }^{(G)} \mathbb{C}_{A} \equiv \chi\left({ }^{(g)} \mathcal{C}_{A}\right), \\
{ }^{(B)} \mathbb{C}_{R} \equiv \chi^{2}\left({ }^{(b)} \mathcal{C}_{R}\right), & { }^{(B)} \mathbb{C}_{A} \equiv \chi\left({ }^{(b)} \mathcal{C}_{A}\right)+\frac{1}{8}(\xi-1)\left({ }^{(\eta)} \mathcal{C}_{A}\right), \\
{ }^{(U)} \mathbb{C}_{R} \equiv \chi^{3}\left({ }^{(u)} \mathcal{C}_{R}\right), & { }^{(U)} \mathbb{C}_{A} \equiv \chi^{2}\left({ }^{(u)} \mathcal{C}_{A}\right) .
\end{array}
$$

Notice that ${ }^{(\eta)} \mathcal{C}_{A}$ is not rescaled as it is associated with the auxiliary variable whose evolution equation was chosen ad hoc. It is possible to define $\eta$ so that it could be treated on an equal footing with the other variables, but as it already serves the purpose required we have not tried to do so. Moreover, as the evolution equations (26) contain at most first radial derivatives of $\eta$ there is no need to introduce a reduction variable to encode $\partial_{r} \eta$ and, consequently, the associated reduction constraint ${ }^{(\eta)} \mathcal{C}_{R}$ is also not required. Direct computation using equation (3) reveals,

$$
\begin{aligned}
&{ }^{(G)} \mathbb{C}_{R}= \frac{\chi}{R^{\prime}} \partial_{r} G+\frac{1}{2 R^{\prime} c_{-}^{r}} G^{+}+\frac{\chi}{2 R^{\prime} c_{+}^{r}} G^{-}-\frac{Q}{2 R^{\prime} \chi c_{-}^{r}} G, \\
&{ }^{(B)} \mathbb{C}_{R}= \frac{\chi}{R^{\prime}} \partial_{r} B-\frac{\chi(\xi-1)}{8 R^{\prime}} \partial_{r} \eta+\frac{1}{2 R^{\prime} c_{-}^{r}} B^{+} \\
&+\frac{\chi}{2 R^{\prime} c_{+}^{r}} B^{-}-\frac{Q}{2 R^{\prime} \chi c_{-}^{r}} B-\frac{R^{2}(\xi-1)}{8 R^{\prime} \chi^{5} c_{+}^{r}} P^{2} \\
&+\frac{1}{16}\left(2 \chi^{\prime}(\xi-1)-2 \chi \xi^{\prime}+\frac{R(\xi-2)}{R^{\prime} \chi c_{-}^{r}}\right) \eta, \\
&(U) \mathbb{C}_{R}=\frac{\chi}{R^{\prime}} \partial_{r} U+\frac{1}{2 R^{\prime} c_{-}^{r}} U^{+}+\frac{\chi}{2 R^{\prime} c_{+}^{r}} U^{-}-\frac{Q}{R^{\prime} \chi c_{-}^{r}} U,
\end{aligned}
$$

and

$$
\begin{aligned}
{ }^{(G)} \mathbb{C}_{A} & =\partial_{A} G-G_{A}, & { }^{(B)} \mathbb{C}_{A}=\partial_{A} B-B_{A}, \\
{ }^{(U)} \mathbb{C}_{A} & =\partial_{A} U-U_{A}, & { }^{(\eta)} \mathcal{C}_{A}=\partial_{A} \eta-\eta_{A},
\end{aligned}
$$

where we have introduced $Q=R+2 R^{\prime} \chi \chi^{\prime} c_{-}^{r}$ to write these expressions succinctly, for the constraints. In the next subsection we fix the asymptotics for $H^{\prime}, R^{\prime}$ and $\chi$. Under those conditions one can verify that $Q \simeq R c_{-}^{r}$.

Discussion: In 16, in a mathematical relativity context, the Gauss curvature of certain two-spheres was taken as an unknown variable in place of a component of the four-dimensional Riemann tensor, the two being related by the Gauss equation. In hindsight our regularization strategy is rather similar, in the sense that a nonlinear change of variables is made to try and derive equations avoiding the worst behaved quantities. For now it is not clear if this method can be applied to arbitrary PDEs satisfying some kind-of weak-null condition, but we do suspect that to be the case.

\section{The compactified asymptotic system}

In this subsection we obtain the asymptotic expressions implied by the evolution equations $(23)-(26)$. Observe that in these expressions neither the compression nor the height functions $R(r)$ and $H(R)$ have been fixed, and we must now do so. In the following we therefore consider $\chi(R), R(r)$ and $H(R)$ with the following asymptotics,

$$
\chi \simeq R, \quad R^{\prime} \simeq R^{n}, \quad H^{\prime} \simeq 1-R^{-n} .
$$

Here $1<n \leq 2$ is a parameter that controls the asymptotic behavior of $R(r)$. Observe that the condition $n>1$ is needed so that $R \rightarrow \infty$ as $r \rightarrow r_{\mathscr{I}}$ for a finite $r_{\mathscr{I}}$. On the other hand, as discussed in [17, $0<n<2$ is required for numerical stability. Near null-infinity the equations of motion then take the form,

$$
\begin{aligned}
\partial_{t} \Psi^{+}= & -\frac{1}{2} \gamma_{2} \Psi^{+}+\mathcal{O}\left(R^{n-2}(\ln R)^{p_{\Psi}}\right), \\
\partial_{t} \Psi^{-}= & -\partial_{r} \Psi^{-}+\gamma_{2}\left(-\partial_{r} \Psi-\frac{1}{2} \Psi^{-}+\mathcal{O}\left(R^{1-n}(\ln R)^{p_{\Psi}}\right)\right) \\
& +\mathcal{O}\left(R^{n-2}(\ln R)^{p_{\Psi}}\right), \\
\partial_{t} \Psi= & \frac{1}{2} \Psi^{-}+\mathcal{O}\left(R^{-1}(\ln R)^{p_{\Psi}}\right), \\
\partial_{t} \Psi_{A}= & \frac{1}{2} \partial_{A} \Psi^{-}+\gamma_{2}\left(\partial_{A} \Psi-\Psi_{A}-\frac{1}{8} p_{\Psi}(\xi-1)\left(\partial_{A} \eta-\eta_{A}\right)\right) \\
& +\mathcal{O}\left(R^{-1}\right), \\
\partial_{t} \eta= & \left(G^{-}\right)^{2}+\mathcal{O}\left(R^{-1}\right), \quad \partial_{t} \eta_{A}=2 G^{-} \partial_{A} G^{-}+\mathcal{O}\left(R^{-1}\right),
\end{aligned}
$$

where $\Psi \in\{G, B, U\}$ with $p_{G}=p_{U}=0$ and $p_{B}=1$. These expressions suggest that, in order to obtain regular expressions at $\mathscr{I}^{+}$it is necessary to assume some decay on $\gamma_{2}$ and restrict the range of the parameter $n$. Taking these considerations into account, setting $\gamma_{2} \simeq R^{-\omega}$ with $\omega>0$ and taking $1<n<2$, the asymptotic system reads

$$
\begin{aligned}
& \partial_{t} \Psi^{+} \simeq 0, \quad \ell \Psi^{-} \simeq 0, \quad \partial_{t} \Psi \simeq \frac{1}{2} \Psi^{-}, \\
& \partial_{t} \Psi_{A} \simeq \frac{1}{2} \partial_{A} \Psi^{-}, \quad \partial_{t} \eta \simeq\left(G^{-}\right)^{2}, \quad \partial_{t} \eta_{A} \simeq 2 G^{-} \partial_{A} G^{-}
\end{aligned}
$$

where $\ell=\partial_{t}+\partial_{r}$. The latter vector corresponds, asymptotically, to the outgoing null direction. To see this, a direct computation using equation $(28)$ shows that

$$
\ell \simeq R^{n} L .
$$

Similarly, the constraints take the form,

$$
\begin{aligned}
& { }^{(\Psi)} \mathbb{C}_{R} \simeq-\Psi^{+}+R^{1-n}\left(\partial_{r} \Psi+\frac{1}{2} \Psi^{-}\right)+\mathcal{O}\left(R^{\left(p_{\Psi}-n\right)}(\ln R)^{p_{\Psi}}\right), \\
& { }^{(\Psi)} \mathbb{C}_{A}=\partial_{A} \Psi-\Psi_{A} .
\end{aligned}
$$

The first of these implies that if the reduction constraints are satisfied, then even if $\partial_{r} \Psi+\frac{1}{2} \Psi^{-} \simeq \mathcal{O}(1)$, a condition weaker than that indicated by the asymptotic system, then $\Psi^{+} \simeq R^{1-n}$, and so it must decay near $\mathscr{I}^{+}$. It follows from the asymptotic system (29) that the choice $n=2$ is out of reach for the bads if we insist on having regular equations for regular unknowns. In the approach discussed in 18, 19 the conformal factor 
is a fixed function of the radial coordinate that regularizes the conformal metric and thus, in our setup, corresponds to the choice $n=2$. Consequently we can only compare our good field with the wave equation in the setup of 20. Although the asymptotic analysis has not been performed for the formulation of [18] nor for the conformal field equations 2, given what we have seen for harmonic gauge the presence of logs in the solutions in those setups is possible too. This point could be addressed by such an analysis for those formulations, which we postpone for future work.

\section{NUMERICAL EVOLUTIONS}

Having given the model and the strategy for regularization we now move on to our numerical implementation. In section III A we discuss the methods employed and the specific data evolved. In section IIIB we present our data.

\section{A. Code overview}

Continuum choices: For the numerical implementation we take the following for the height and compress functions,

$$
H^{\prime}=1-\frac{1}{R^{\prime}}, \quad R(r)=\frac{r}{\Omega^{\frac{1}{n-1}}}, \quad \Omega(r)=1-\frac{r^{2}}{l^{2}},
$$

so that $\mathscr{I}^{+}$is located at $r=l$, and always set $l=1$. Observe that the above choice for the height function implies that,

$$
c_{+}^{r}=1 \quad c_{-}^{r}=-\frac{1}{2} R^{-n}+\mathcal{O}\left(R^{-2 n}\right) .
$$

The latter ensures that outgoing pulses propagate without distortion as they move towards $\mathscr{I}^{+}$. For the rescaling function $\chi$ and the damping parameter we choose

$$
\chi=\sqrt{1+R^{2}}, \quad \gamma_{2}=\frac{\gamma}{\chi} .
$$

This choice satisfies the conditions of equation 28 so that in the asymptotic limit one recovers equations (30). The reason for setting $\chi=\sqrt{1+R^{2}}$ is to avoid introducing unnecessary singular terms at the origin $R=0$. As initial data we set each of the raw fields $g, b$ and $u$, to

$$
a e^{-\delta\left(R-R_{0}\right)^{2}}+a e^{-\delta\left(R+R_{0}\right)^{2}},
$$

keeping the freedom to adjust the amplitude, width and offset parameters $a, \delta, R_{0}$ separately for each field. The values for the regularized fields $G, B$ and $U$ are then computed by taking derivatives and/or applying the change of variable in the obvious manner. The auxiliary variable $\eta$ is taken to vanish initially.
Numerical setup: Our experiments have been performed in a one-dimensional code that uses very standard methods, and shares the same basic infrastructure as that used for the spherically symmetric hyperboloidal evolutions in [18, 19]. We now give a quick overview of these methods. The method of lines is employed for time integration, and is performed with a fourth order accurate Runge-Kutta. To approximate spatial derivatives we use second order centered finite differences. We made this choice because the small stencil makes the propagation of noise potentially slower than with higher order finite differences or spectral methods. The only subtlety in the implementation is that, because the evolution equations (4) were written in spherical polar coordinates the equations $(23)-(26)$ contain divergent terms at the origin. Since we are performing spherically symmetric evolutions the $1 / R^{2}$ coefficient of $q^{A B} \nabla_{A} \Psi_{B}$ is not problematic, but the $1 / R$ terms present in the source terms $S_{\Psi}$ (displayed in Appendix A require special attention. Our solution is to use Evans method [21] as discussed in 22 in the context of summation by parts discretizations of the wave equation in spherical symmetry. In the latter it is shown that given a system of equations of the form,

$$
\partial_{t} \psi=\partial_{r} \pi, \quad \partial_{t} \pi=\partial_{r} \psi+\frac{p}{r} \psi
$$

the spatial derivatives can be discretized as,

$$
\partial_{t} \psi=h^{-1} D \pi, \quad \partial_{t} \pi=h^{-1} \tilde{D} \psi,
$$

where $h \equiv \Delta r$ is the grid spacing and the difference operators $D$ and $\tilde{D}$ are given by

$$
\begin{aligned}
h^{-1} \tilde{D} \psi & =(p+1) \frac{r_{i+1}^{p} \psi_{i+1}-r_{i-1}^{p} \psi_{i-1}}{r_{i+1}^{p+1}-r_{i-1}^{p+1}}, \\
h^{-1} D \pi & =\frac{\pi_{i+1}-\pi_{i-1}}{2},
\end{aligned}
$$

where $\psi_{i}(t)$ and $\pi_{i}(t)$ are grid functions approximating $\psi(t, r)$ and $\pi(t, r)$ on a grid $r_{i}$. We work always with a non-staggered grid so that there are gridpoints both directly at the origin and at $\mathscr{I}^{+}$. In order to rewrite equations (23)- 26) in a form in which the discretization (37) can be applied, we define $\Xi$ according to,

$$
\frac{1}{R}=\frac{1}{R^{\prime}}\left(\frac{1}{r}-\frac{\Xi^{\prime}}{\Xi}\right)
$$

which can be used to rewrite the terms in $S_{\Psi}$ with contain $1 / R$ and then exploit the aforementioned discretization to absorb the singular behavior at the origin with $D$ and $\tilde{D}$. Although these equations are regular everywhere (including at $\mathscr{I}^{+}$), the coefficients in these equations are in general of the form $Q_{m}(R) / Q_{s}(R)$ with $m \leq s$ or $\ln (R) Q_{m}(R) / Q_{s}(R)$ with $m<s$ where $Q_{n}(R)$ denotes a polynomial in $R$ of degree $n$. Thus to avoid evaluating numerically the quotient of two large numbers careful algebraic manipulations are required. In practice, one can opt also to substitute $R(r)$ explicitly. To manage the inner boundary we define ghostzones, which are populated 
from the bulk variables using the known parity of the raw $g, b$ and $u$ fields and their derivatives. By construction no physical boundary conditions are needed at $\mathscr{I}^{+}$, but derivatives must still be approximated. To make that possible we extrapolate the evolved fields from the bulk into one ghostzone at fourth order and use the standard spatial operators all of the way out to the boundary point. The final ingredient in our method is the use of Kreiss-Oliger dissipation 23

$$
\sigma h^{3} D_{+}^{2} D_{-}^{2} / 16
$$

with $D_{ \pm}$the standard forward and backward differencing operators, which, as used in [24], is added to each of the evolution equations to reduce high-frequency noise. An exception is the $\eta$ variable, which is treated differently because no spatial derivatives of this quantity are present anywhere in the system, and experimentally we find that this leads to a misleading third order convergent feature at the resolutions we employ when dissipation is used on the variable. At the outer boundary we use the same extrapolation mentioned above to fill the additional point in the dissipation stencil.

\section{B. Results with the GBU model}

Basic dynamics: We begin with a description of the basic dynamics of the system. To give ball-park figures we find that 200 spatial points and a CFL factor of $1 / 2$ are typically sufficient to provide well-resolved solutions that appear smooth in space and time given our initial data, and is also sufficient to see convergence experimentally, see Fig. 3. We work always with the dissipation parameter $\sigma=2 / 100$, see $(39)$. Following $(35)$ and placing identical data centered at the origin $R_{0}=0$ with width parameter $\delta=1$ and amplitude $a=1 / 100$ in each of the raw fields $g, b, u$, and trivial initial data for the auxiliary variable $\eta$, we see the expected behavior. The data for each field splits into two pulses. The first of these propagates directly out to null-infinity, whereas the second appears to oscillate briefly at the origin first. A brief comparison with results of an older code for the plain-wave equation in first order in time, second order in space form, written in the same infrastructure, against the good field $g$ reveals comparable results, again giving us confidence in the numerics (taking $n=2$ as mentioned earlier). In figure 1 we plot snapshots of the solution for the bad and ugly fields given the aforementioned initial data. The ugly field $u$ is decoupled from the rest of the system, but behaves in a qualitatively similar manner. At late times we see a hump near $\mathscr{I}^{+}$in the rescaled ugly field $U$ that appears to decay slowly. The important first result here is that the behavior predicted by the asymptotic system and hoped for in our regularization is realized; our evolved fields and their derivatives are finite, and the equations of motion are explicitly regular. The basic dynamics described above does not change if one adds a small offset $R_{0} \neq 0$ or gives different widths $\delta$ for each of the raw fields $g, b, u$.

Behavior of the reformed bad and auxiliary fields: For our particular model there is no question of finite time blow-up, so any explosion of the data must be caused by a failure of the numerical method; we see no such blowup. That said, as we increase the amplitude of the $g$ field or give non-trivial initial data for $\eta$, we see both that the distortion in $B$ increases and, obviously, that $\eta$ grows, indicating that the original $b$ variable is picking up a log-term as expected. In figure 2 we display a spacetime plot of the outgoing radiation-field $B^{-}$. Since the $g$ field rapidly propagates out through $\mathscr{I}^{+}$, we find that $\eta$ reaches a fixed, non-zero end-state rather quickly.

Constraint damping: One potential weakness of the present formulation is that for regularity of the field equations we have to suppress the constraint damping parameter $\gamma_{2}$ like $1 / R$ near $\mathscr{I}^{+}$. One might therefore worry that enforcing strong-damping $\gamma_{2}=O(1)$ near the origin would result in small violations near the origin and large violations near $\mathscr{I}^{+}$, which could generate large gradients and hence large errors, even if the scheme were converging reliably. To investigate this we have compared evolutions with and without constraint damping switched on, and find that within the run-times considered $t \simeq 100$, such problems do not manifest. We think it may be possible to adjust the present constraint damping scheme to damp violations on outgoing pulses in such a way that we could maintain $O(1)$ constraint damping parameters, but since doing so would require re-engineering the entire scheme, and we presently do not see a pressing need for such a modification, we have not pushed this line of inquiry further.

Convergence: In the future we hope that a regularization similar to that employed here will be useful in gravitational wave applications. Such work requires meaningful error-estimates. Our experiments can therefore only be considered a success if clean convergence can be experimentally achieved. To investigate this we performed a set of runs in which we start from the grid-setup mentioned above and then doubled resolution several times, keeping the remaining parameters fixed. These tests were furthermore performed with several different choices for the initial data parameters. In figure 3 we display two of the ensuing convergence plots. In the first we show that perfect pointwise convergence for the outgoing radiation fields $B^{-}$and $U^{-}$is attained at $\mathscr{I}^{+}$. The second shows that good second order convergence is obtained in the norm of the full solution as resolution is increased. In the latter we do notice a gradual drift away from second order at late times, but this effect is suppressed as resolution increases, so does not appear to be a problem in principle. It may be that by adjusting the specifics of our outer boundary treatment this behavior can be improved. So far we have not done so systematically however. We have also examined the constraint violations (27) and find perfect second order convergence in all quantities. 

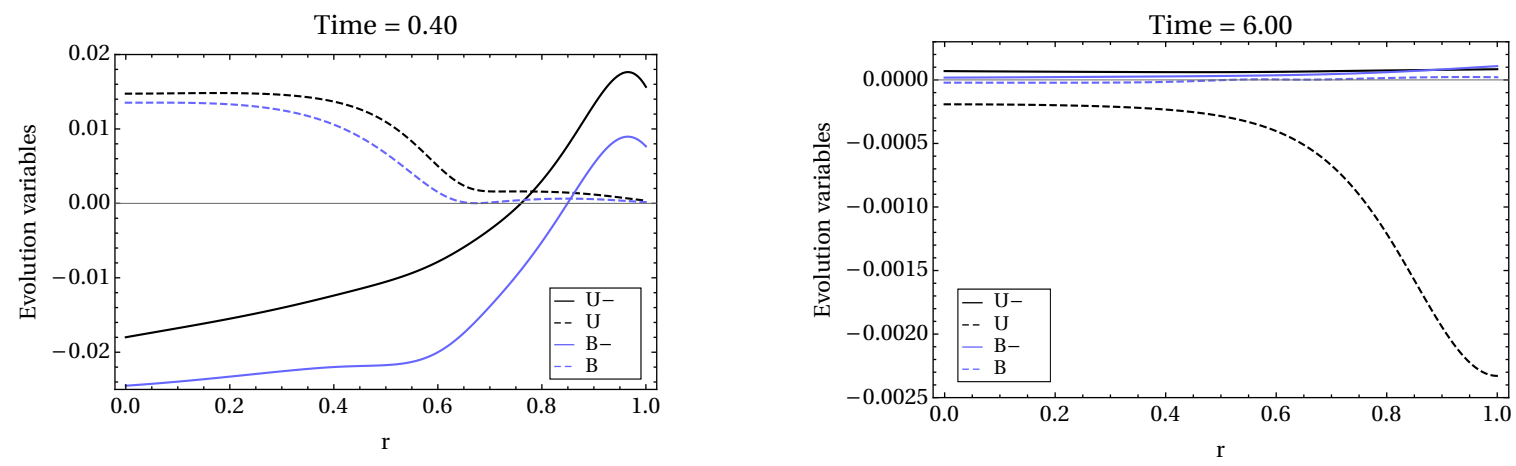

FIG. 1: In these plots we display snapshots of the solution for the regularized ugly $U^{-}, U$ and bad $B^{-}, B$ fields at fixed times, against the compactified radial coordinate $r$. In the left panel the time $t=0.4$ was chosen as it corresponds roughly with the maximum of the radiation field $B^{-}$at null-infinity (compare with Fig. 2). The right panel serves to demonstrate the decay of the fields after the initial pulse leaves the domain. Note the difference in scale.

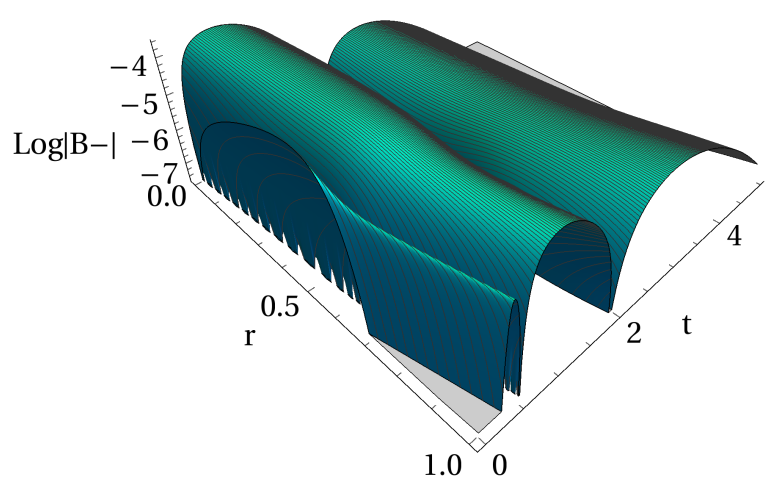

FIG. 2: Here we plot the reformed bad field $B^{-}$as a function of spacetime, again against the compactified radial coordinate $r$. The take home message is that the initial pulse exits the domain cleanly, with no visible numerical reflection, leaving behind a low-frequency feature in space that gradually decays.

\section{CONCLUSIONS}

Continuing towards a robust treatment of future nullinfinity in numerical relativity we considered a semilinear system of wave equations. The system was constructed with the nonlinear structure of the field equations of GR in harmonic gauge in mind, and has three different types of fields. We call these the good, the bad and the ugly. Of these, the good fields have fall-off near null-infinity like solutions of the wave equation, whereas the ugly fields decay faster. Finally the bad fields decay worse that solutions to the wave equation by a logarithm in $R$. The main accomplishment in this paper was to give a reformulation of the equations that delivers regular equations for regular, generically non-decaying, variables on compactified hyperboloidal slices. The regularization strategy is to rescale all of the wave fields as aggressively as possible and then define new fields to subtract out any potential logarithmic, or perhaps harsher, divergences. The crucial technical tool was the use of an asymptotic expansion, which allowed us to discard irrelevant terms. In our model this meant the introduction of the $\eta$ field, and by analogy in GR will mean integrating up the square of the Bondi news function near null-infinity. Remarkably the asymptotic expansion of the regularized system is identical to that of a set of decoupled wave equations. With the regularization in hand we presented a set of numerical evolutions in which we demonstrated perfect pointwise and norm convergence over the entire computational domain, which included null-infinity explicitly. Future tests of the model will be performed using the bamps pseudospectral numerical relativity code [25, 26], which presents a more subtle challenge because spectral methods may more susceptible to any lingering lower-order log-terms. In forthcoming work we will present a regularization of GR following the same approach.

\section{Acknowledgments}

We are grateful to Sukanta Bose, Bernd Brügmann, Rodrigo Panosso Macedo, Isabel Suárez Fernández and Juan Valiente Kroon for helpful discussions or comments on the manuscript. EG, DH and AVV also gratefully acknowledge the support and hospitality offered by IUCAA, Pune, where part of this work was completed. Many of our derivations were performed in xAct 27] for Mathematica. The notebooks are available at 28. The work was partially supported by the FCT (Portugal) IF Program IF/00577/2015, PTDC/MATAPL/30043/2017, the European Research Council Consolidator Grant 647839, the GWverse COST action Grant No. CA16104, and under the PhD researcher Decree-Law no. 57/2016 of August 29 (Portugal), IUCAA's NRTT grant and the UGC of India. 

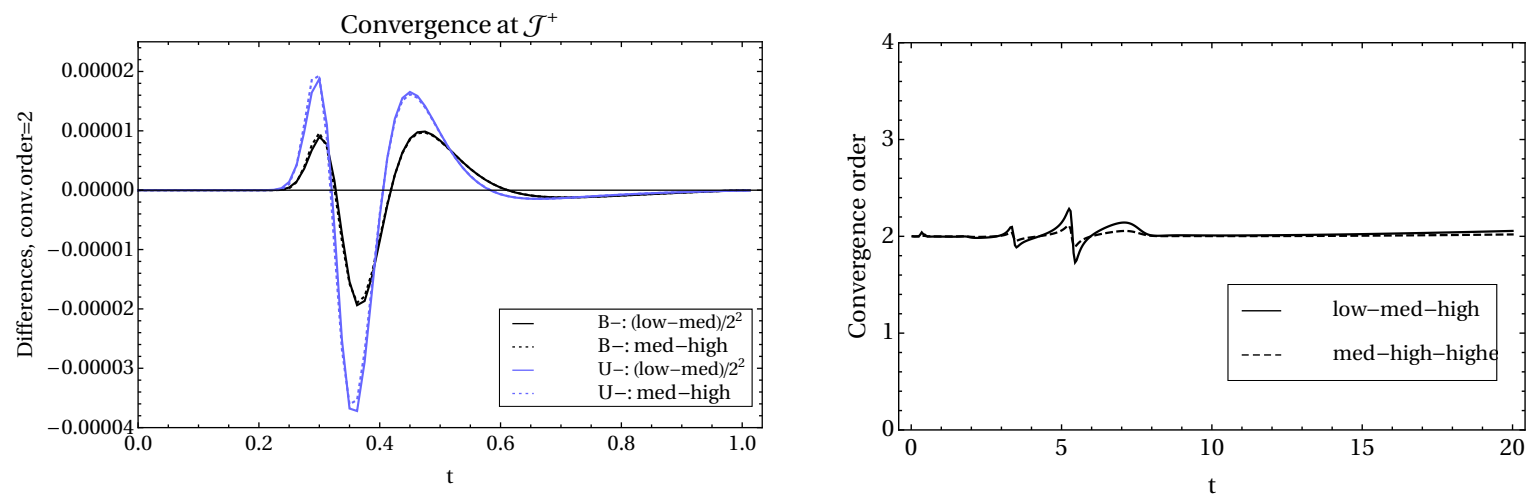

FIG. 3: To judge whether or not the numerical method is working reliably we perform convergence tests as usual. In going from low to medium and medium to high resolution we doubled resolution. In the left panel we demonstrate that the outgoing radiation fields $U^{-}$and $B^{-}$converge perfectly at second order at null-infinity as a function of time, consistent with our discretization. In the second panel we display the experimental convergence rate in the norm of the difference of the solutions as a function of time. At first glance it seems that there is a problem abound $t=4$ and $t=6$, but, as can be seen by the second curve, this effect converges away.

\section{Appendix A: Source terms in the GBU model}

The source terms $S_{\Psi}$ of equation 26 are expressions polynomials in the fields $\Phi$ whose coefficients are regular functions of $R$. Using the notation of Section IIC] the sources for $G^{+}$and $G^{-}$are given by

$$
\begin{aligned}
& S_{G^{+}}=-\frac{R^{\prime} c_{-}^{r}}{R \chi}(R-\chi)(R+\chi) G^{-}+\frac{2 R^{\prime} c_{-}^{r}}{\chi^{2}}\left(\chi-R \chi^{\prime}\right) G \\
& +\frac{\gamma_{2}}{2}\left(\frac{1}{\chi}\left(R+2 R^{\prime} \chi \chi^{\prime} c_{-}^{r}\right) G-G^{+}-\chi\left(1+2 R^{\prime} c_{-}^{r}\right) G^{-}\right) \\
& +\frac{R^{\prime} c_{-}^{r}}{R \chi}\left(2 R \chi^{\prime}-\chi\right) G^{+}
\end{aligned}
$$

and

$$
\begin{aligned}
S_{G^{-}} & =\frac{R^{\prime} c_{+}^{r}}{R \chi} G^{+}+\frac{R^{\prime} c_{+}^{r}}{R \chi}\left(R \chi^{\prime}-\chi\right) G^{-}-\frac{R^{\prime} c_{+}^{r}}{\chi^{2}} G \\
& +\frac{\gamma_{2}}{2}\left(\frac{1}{\chi}\left(2 R^{\prime} c_{+}^{r}-1\right) G^{+}-G^{-}\right. \\
& \left.+\frac{1}{\chi^{2}}\left(R-2 R^{\prime} R c_{+}^{r}+2 R^{\prime} \chi \chi^{\prime} c_{+}^{r}\right) G\right) .
\end{aligned}
$$

For the $B^{+}$and $B^{-}$fields we have,

$$
\begin{aligned}
& S_{B^{+}}=\frac{1}{8 \chi^{7}} F_{1}^{+}\left(\chi^{2} G^{-}-\chi G^{+}+R G\right)^{2}-\frac{R^{\prime} c_{-}^{r}}{8 \chi^{2}} F_{3}^{+} \eta \\
& +\frac{\gamma_{2}}{2}\left(\frac{1}{8 \chi} F_{2}^{+} \eta-B^{+}+\frac{1}{\chi}\left(R+2 R^{\prime} \chi \chi^{\prime} c_{-}^{r}\right) B\right. \\
& \left.+\frac{R^{2} c_{-}^{r}}{4 \chi^{5} c_{+}^{r}}(\xi-1)\left(\chi^{2} G^{-}-\chi G^{+}+R G\right)^{2}-\frac{\chi c_{-}^{r}}{c_{+}^{r}} B^{-}\right) \\
& -\frac{R^{\prime} c_{-}^{r}}{R \chi}(R-\chi)(R+\chi) B^{-}+\frac{2 R^{\prime} c_{-}^{r}}{\chi^{2}}\left(\chi-R \chi^{\prime}\right) B \\
& +\frac{R^{\prime} c_{-}^{r}}{R \chi}\left(2 R \chi^{\prime}-\chi\right) B^{+}, \\
& S_{B^{-}}=\left(\frac{R^{\prime} R}{2 \chi^{6}} F_{1}^{-}-\frac{\gamma_{2} R^{2} c_{-}^{r}}{4 \chi^{4} c_{+}^{r}}(\xi-1) F_{7}^{-}\right) G G^{-}+\frac{R^{\prime} c_{+}^{r}}{8 \chi^{2}}(\xi-2) \eta \\
& +\frac{\gamma_{2} c_{+}^{r} F_{9}^{-}}{16 \chi^{2} c_{-}^{r}} \eta+\left(\frac{R^{\prime} c_{+}^{r}}{R \chi}\left(R \chi^{\prime}-\chi\right)-\frac{\gamma_{2}}{2}\right) B^{-}+\left(\frac{R^{\prime} c_{+}^{r}}{R \chi}-\frac{\gamma_{2} c_{+}^{r}}{2 \chi c_{-}^{r}}\right) B^{+} \\
& +\left(\frac{\gamma_{2} R^{2} c_{-}^{r}}{8 \chi^{2} c_{+}^{r}}(\xi-1)\left(2 R^{\prime} c_{+}^{r}-3\right)-\frac{R^{\prime}}{4 \chi^{4}} F_{3}^{-}\right)\left(G^{-}\right)^{2}
\end{aligned}
$$

$$
\begin{aligned}
& +\left(\frac{\gamma_{2} R^{2}}{8 \chi^{4}}(\xi-1)\left(4 R^{\prime} c_{+}^{r}-3\right)-\frac{R^{\prime}}{4 \chi^{6}}\left(F_{4}^{-}+F_{5}^{-}\right)\right)\left(G^{+}\right)^{2} \\
& -\left(\frac{R^{\prime} c_{+}^{r}}{\chi^{2}}-\frac{\gamma_{2}}{2 \chi^{2}}\left(R-2 R^{\prime} R c_{+}^{r}+2 R^{\prime} \chi \chi^{\prime} c_{+}^{r}\right)\right) B \\
& +\left(\frac{\gamma_{2} R^{3} c_{-}^{r} F_{8}^{-}}{8 \chi^{6} c_{+}^{r}}(\xi-1)-\frac{R^{\prime} R^{2}}{4 \chi^{8}}\left(F_{4}^{-}+F_{5}^{-}-4 R^{\prime} R \chi^{2} c_{-}^{r} c_{+}^{r}\right)\right) G^{2} \\
& +\left(\frac{R^{\prime} R}{2 \chi^{7}}\left(F_{4}^{-}+F_{5}^{-}-2 R^{\prime} R \chi^{2} c_{-}^{r} c_{+}^{r}\right)-\frac{\gamma_{2} R^{2} c_{-}^{r} F_{6}^{-}}{4 \chi^{5} c_{+}^{r}}(\xi-1)\right) G G^{+} \\
& -\left(\frac{R^{\prime} F_{2}^{-}}{2 \chi^{5}}+\frac{\gamma_{2} R^{2} c_{-}^{r}}{4 \chi^{3} c_{+}^{r}}(\xi-1)\left(3-6 R^{\prime} c_{+}^{r}+4 R^{\prime 2}\left(c_{+}^{r}\right)^{2}\right)\right) G^{-} G^{+},
\end{aligned}
$$

where the coefficients $F^{ \pm}$are functions of $R$ only. The detailed expressions for these are given in the following lists. The '+' quantities are,

$$
\begin{aligned}
F_{1}^{+}= & R^{3}-2 R^{\prime} R^{3} c_{-}^{r}+2 R^{\prime} R \chi^{2} c_{-}^{r}+2 R^{\prime} \chi^{3} c_{-}^{r} \\
& +2 R^{\prime} R^{3} \xi c_{-}^{r}-2 R^{\prime} R \chi^{2} \xi c_{-}^{r}, \\
F_{2}^{+}= & 2 R-R \xi+2 R^{\prime} \chi \chi^{\prime} c_{-}^{r}-2 R^{\prime} \chi \chi^{\prime} \xi c_{-}^{r}+2 R^{\prime} \chi^{2} \xi^{\prime} c_{-}^{r}, \\
F_{3}^{+}= & -4 \chi+5 R \chi^{\prime}+2 \chi \xi-2 R \chi^{\prime} \xi .
\end{aligned}
$$

The '-' quantities are,

$$
\begin{aligned}
F_{1}^{-}= & \chi^{3} c_{+}^{r}-2 R^{3}(\xi-1) c_{-}^{r}\left(R^{\prime} c_{+}^{r}-1\right) \\
& +R \chi^{2}(\xi-1) c_{-}^{r}\left(8 R^{\prime} c_{+}^{r}-3\right) \\
& -R^{2} \chi\left(\chi \xi^{\prime} c_{+}^{r}+\chi^{\prime}(\xi-1) c_{-}^{r}\left(6 R^{\prime} c_{+}^{r}-1\right)\right), \\
F_{2}^{-}= & \chi^{3} c_{+}^{r}-3 R \chi^{2}(\xi-1) c_{+}^{r}-2 R^{3}(\xi-1) c_{-}^{r}\left(R^{\prime} c_{+}^{r}-1\right) \\
& -R^{2} \chi\left(\chi \xi^{\prime} c_{+}^{r}+\chi^{\prime}(\xi-1) c_{-}^{r}\left(6 R^{\prime} c_{+}^{r}-1\right)\right), \\
F_{3}^{-}= & 2 R^{3}(\xi-1) c_{-}^{r}+\chi^{3} c_{+}^{r}-R^{2} \chi\left(\chi^{\prime}-\chi^{\prime} \xi+\chi \xi^{\prime}\right) c_{+}^{r} \\
& +R \chi^{2}(\xi-1) c_{-}^{r}\left(2 R^{\prime} c_{+}^{r}-3\right), \\
F_{4}^{-}= & -R \chi\left(R \chi \xi^{\prime}-10 R^{\prime} \chi(\xi-1) c_{-}^{r}+10 R^{\prime} R \chi^{\prime}(\xi-1) c_{-}^{r}\right) c_{+}^{r}, \\
F_{5}^{-}= & -3 R \chi^{2}(\xi-1) c_{-}^{r}+R^{2} \chi \chi^{\prime}(\xi-1) c_{-}^{r}+\chi^{3} c_{+}^{r} \\
& +2 R^{3}(\xi-1) c_{+}^{r}, \\
F_{6}^{-}= & 4 R^{\prime} \chi \chi^{\prime} c_{+}^{r}\left(R^{\prime} c_{+}^{r}-1\right)+R\left(10 R^{\prime} c_{+}^{r}-8 R^{\prime 2}\left(c_{+}^{r}\right)^{2}-3\right), \\
F_{7}^{-}= & 4 R^{\prime} \chi \chi^{\prime} c_{+}^{r}\left(R^{\prime} c_{+}^{r}-1\right)+R\left(6 R^{\prime} c_{+}^{r}-4 R^{\prime 2}\left(c_{+}^{r}\right)^{2}-3\right),
\end{aligned}
$$


$F_{8}^{-}=8 R^{\prime} \chi \chi^{\prime} c_{+}^{r}\left(R^{\prime} c_{+}^{r}-1\right)+R\left(10 R^{\prime} c_{+}^{r}-8 R^{\prime 2}\left(c_{+}^{r}\right)^{2}-3\right)$,
$F_{9}^{-}=-R(\xi-2)+2 R^{\prime} \chi\left(\chi^{\prime}-\chi^{\prime} \xi+\chi \xi^{\prime}\right) c_{-}^{r}$.

Finally, the sources for $U^{ \pm}$are given by,

$$
\begin{aligned}
S_{U^{+}} & =\frac{R^{\prime} c_{-}^{r}}{R \chi}(\chi-R)(2 R+\chi) U^{-}+\frac{2 R^{\prime} c_{-}^{r}}{\chi^{2}}\left(2 \chi-R-2 R \chi^{\prime}\right) U \\
& -\frac{\gamma_{2}}{2}\left(U^{+}+\chi\left(1+2 R^{\prime} c_{-}^{r}\right) U^{-}-\frac{2}{\chi}\left(R+2 R^{\prime} \chi \chi^{\prime} c_{-}^{r}\right) U\right) \\
& +\frac{R^{\prime}}{R \chi c_{-}^{r}}\left(R-\chi+3 R \chi^{\prime}\right) U^{+},
\end{aligned}
$$

and,

$$
S_{U^{-}}=\frac{2 R^{\prime} c_{+}^{r}}{\chi^{3}}(R-\chi) U-\frac{R^{\prime}}{R \chi^{2} c_{+}^{r}}(R-\chi) U^{+}
$$

$$
\begin{aligned}
& -\frac{R^{\prime} c_{+}^{r}}{R \chi}\left(R+\chi-2 R \chi^{\prime}\right) U^{-} \\
& -\frac{\gamma_{2}}{2}\left(U^{-}-\frac{1}{\chi}\left(2 R^{\prime} c_{+}^{r}-1\right) U^{+}\right. \\
& \left.-\frac{2}{\chi}\left(R-2 R^{\prime} R c_{+}^{r}+2 R^{\prime} \chi \chi^{\prime} c_{+}^{r}\right) U\right) .
\end{aligned}
$$

[1] R. Penrose, Phys. Rev. Lett. 10, 66 (1963).

[2] H. Friedrich, Proc. Roy. Soc. London A 375, 169 (1981)

[3] H. Friedrich, Proc. Roy. Soc. London A 378, 401 (1981).

[4] A. Zenginoglu, Class. Quant. Grav. 25, 195025 (2008), 0808.0810 .

[5] J. Frauendiener, Living Rev. Relativity 7 (2004).

[6] D. Hilditch, E. Harms, M. Bugner, H. Rüter, and B. Brügmann, Class. Quant. Grav. 35, 055003 (2018), 1609.08949.

[7] D. Hilditch (2015), 1509.02071.

[8] E. Gasperin and D. Hilditch, Class. Quant. Grav. 36, 195016 (2019), 1812.06550.

[9] H. Lindblad and I. Rodnianski, Comptes Rendus Mathematique 336, 901 (2003), ISSN 1631073X, URL http://www.sciencedirect.com/science/ article/pii/S1631073X03002310

[10] J. Keir, ArXiv e-prints (2018), 1808.09982.

[11] J.-A. Valiente-Kroon, Conformal Methods in General Relativity (Cambridge University Press, Cambridge, 2016).

[12] S. Klainerman, Communications on Pure and Applied Mathematics 33, 43 (1980).

[13] S. Klainerman, in Nonlinear systems of partial differential equations in applied mathematics, Part 1 (Santa Fe, N.M., 1984) (Amer. Math. Soc., Providence, RI, 1986), vol. 23 of Lectures in Appl. Math., pp. 293-326.

[14] D. Christoudolou, Communications in Mathematical Physics 105, 337 (1986).

[15] L. Lindblom, M. A. Scheel, L. E. Kidder, R. Owen, and O. Rinne, Class. Quant. Grav. 23, S447 (2006), gr- qc/0512093

[16] J. Luk, J. Am. Math. Soc. 31, 1 (2018), 1311.4970.

[17] G. Calabrese, C. Gundlach, and D. Hilditch, Class.Quant.Grav. 23, 4829 (2006), gr-qc/0512149.

[18] A. Vañó-Viñuales, S. Husa, and D. Hilditch, Class. Quant. Grav. 32, 175010 (2015), 1412.3827.

[19] A. Vañó-Viñuales and S. Husa, Class. Quant. Grav. 35, 045014 (2018), 1705.06298.

[20] A. Vañó-Viñuales, Ph.D. thesis, U. Iles Balears, Palma (2015), 1512.00776, URL http://inspirehep. net/record/1407828/files/arXiv:1512.00776.pdf.

[21] C. R. Evans, Ph.D. thesis, University of Texas at Austin (1984).

[22] C. Gundlach, J. M. Martin-Garcia, and D. Garfinkle, Class. Quant. Grav. 30, 145003 (2013), 1010.2427.

[23] H. O. Kreiss and J. Oliger, Methods for the approximate solution of time dependent problems (GARP publication series No. 10, Geneva, 1973).

[24] M. C. Babiuc et al., Class. Quant. Grav. 25, 125012 (2008), 0709.3559.

[25] B. Brügmann, J. Comput. Phys. 235, 216 (2013), 1104.3408.

[26] D. Hilditch, A. Weyhausen, and B. Brügmann, Phys. Rev. D93, 063006 (2016), 1504.04732.

[27] J. M. Martín-García, xAct: tensor computer algebra. (2017), http://www.xact.es/

[28] https://centra.tecnico.ulisboa.pt/ hilditch/ WeakNullToy.tgz 